\title{
Phase Synchronization and Coherence Analysis between ECG \& Arterial Blood Pressure
}

\author{
Nikhil Pachauri \\ Department of Instrumentation \& control \\ Engineering NIT \\ Jalandhar.
}

\author{
Dipak Kumar Mishra \\ Department of Instrumentation \& control \\ Engineering NIT \\ Jalandhar.
}

\begin{abstract}
Phase synchronization is an important phenomenon of nonlinear dynamics and has recently received much scientific attention. Phase synchronization is a type of synchronization reflecting mutual adjustment of rhythms of self-sustained oscillatory systems. Time-varying phase synchrony is an important bivariate measure that quantifies the dynamics between non-stationary signals and has been widely used in many applications including chaotic oscillators in physics and multichannel electroencephalography recordings in neuroscience. In this paper we investigate that phase synchronization between electrocardiogram and arterial blood pressure was used in order to find the interaction between these two signals. With the help of coherence phase we are able to tell which signal is lagging and which signal is leading.
\end{abstract}

\section{Keywords}

Biomedical Engineering, phase synchronization, coherence

\section{INTRODUCTION}

Biomedical Engineering is the study and application of engineering principle and techniques to the medical field. It is concerned with development and manufacture of prostheses, medical devices, drugs and other therapies. This field encompasses biomedical instrumentation, bio-materials, biomechanics, medical imaging.

A well-known common feature of oscillatory systems and Biological oscillators, in particular, are their ability to synchronize. Entrainment of periodic (also noisy) selfsustained oscillators by external periodic force, or mutual synchronization of several such oscillators is well understood [1-5], and this theoretical knowledge is widely used in experimental studies and in the modeling of interaction between different physiological (sub) systems. The examples range from the modeling of the heart in the pioneering paper of van der Pol and van der Mark [6] to investigation of the circadian rhythm phase locking of respiration with mechanical ventilator. Phase locking of chicken embryonic heart cells with external stimuli and interaction of sinus node with ectopic pacemakers [5], synchronization of oscillations of human insulin secretion and glucose infusion [7], and synchronization of heart rate by external audio or visual stimuli [8].

In the literature [9] they have used the concept of phase synchronization in order to find out the relationship between intracranial pressure and Arterial blood pressure.

In other study [10], the cortical functioning from EEG signal at different sleep stages taken from different pair of electrodes has been analyzed. Phase coupling around $0.1 \mathrm{~Hz}$ between cardiovascular (de) oxy hemoglobin oscillation using cross spectral method [11].
In this work we propose that with the help of phase synchronization we can find the interaction between ECG and Arterial blood pressure. Based on Hilbert Transform, the phase synchronization is calculated. With coherence phase we are able to tell which signal is lagging and which signal is leading.

\section{METHOD AND MATERIAL}

We performed noninvasive examinations with eight healthy volunteers 21 to 27 years. The subjects were laying at rest and no constraints like paced respiration or mental exercising were used. The electrocardiogram (ECG) was registered by standard leads using MP100 Data Acquisition with softwarecontrolled Amplifiers and simultaneously blood pressure was measured by a NIBP100B (Fusion) Noninvasive BP System The duration of each record is 30 minutes. All signals were digitized with $1000-\mathrm{Hz}$ sampling rate and 12-bit resolution. For the analysis of the heart rate the times of $\mathrm{R}$ peaks in the ECG were extracted by a semiautomatic algorithm with manual correction. Only data sets without extra systoles are used for the subsequent analysis. The human cardiovascular systems and blood pressure do not act independently; their interrelation is rather complex and still remains a subject of physiological research.

\subsection{Coherence and Phase Synchronization Analysis}

Coherence is the degree of relationship or association of frequency spectra between the ECG and Arterial blood pressure signals at a particular frequency. The spectral content for each lead is highly similar regardless of the lead configuration, although the actual energy at each frequency may differ. The magnitude squared coherence estimate between two signals x (ECG Signal) and y (Blood Pressure Signal), is

$$
\operatorname{Coh}_{x y}(f)=\frac{\left|C S_{x y}(f)\right|}{\sqrt{S_{x x}(f) \times S_{y y}(f)}}
$$

Where $\operatorname{Coh}_{x y}(f)$ is the magnitude square coherence between ECG and Arterial blood pressure, coherence phase is given as

$$
\theta(f)=\tan ^{-1}\left\{\frac{\operatorname{Im}\left(C S_{x y}\right)}{\operatorname{Re}\left(C S_{x y}\right)}\right\}
$$

Where $S_{x x}(f)$ is the power spectral estimate of $\mathrm{x}$ (ECG Signal)

$S_{y y}(f)$ is the power spectral estimate of y (Arterial blood pressure Signal), and $C S_{x y}(f)$ is the cross power spectral estimate of $\mathrm{x}$ and $\mathrm{y}$. Coherence is a function of frequency with $\operatorname{Coh}_{x y}(f)$ ranging between 0 and 1 and indicates how well signal $\mathrm{x}$ corresponds to signal $\mathrm{y}$ at each frequency [12] 
To investigate the interaction between the oscillating process in arterial blood pressure and ECG is commonly characterized by phase synchronization measure based on Hilbert transform. Firstly we have to calculate the instantaneous phase of these two signals in order to quantify phase synchronization. To calculate the phase of heart rate the analytic signal $\varepsilon(t)$ [13] is constructed for the signal $\mathrm{s}(\mathrm{t})$ obtained as a result of band pass filtration of R-R intervals for different frequency band. The signal $\varepsilon(\mathrm{t})$ is a complex function of time defined as

$$
\varepsilon(t)=s(t)+i \epsilon(t)=A(t) \exp (i \emptyset(t))
$$

Where $A(t)$ and $\varnothing(t)$ are respectively the amplitude and the phase of the analytic signal, and function $\epsilon(t)$ is the Hilbert transform of $s(t)$,

$$
\epsilon(t)=\frac{1}{\pi} P \cdot V \cdot \int_{-\infty}^{+\infty} \frac{s(\tau)}{t-\tau} d \tau
$$

Where P.V. means that the integral is taken in the sense of the Cauchy principal value. Phase $\emptyset(t)$ is defined from (3)

$$
\emptyset(\mathrm{t})=\tan ^{-1}(\epsilon(t) / s(t))
$$

In a similar way the phase of the blood pressure is calculated for different frequency band.

To detect synchronization between blood pressure and ECG the phase difference

$$
\varphi_{m n}=n \emptyset_{1}-m \emptyset_{2}
$$

is calculated where, $\varphi_{m n}$ is the generalized phase difference and $m: n$ is the locking ratio. In the above case, m periods of the first system (represented by $\emptyset_{1}$ ) correspond to $\mathrm{n}$ periods of the second system. Where appropriate, the subscripts $\mathrm{m}, \mathrm{n}$ is dropped to simplify the notation. In the rest of this paper, the term phase difference will be interchangeably used with the term generalized phase difference as m:n phase synchronization is explicitly considered in this work.

The presence of $1: 1$ phase synchronization is defined by the condition

$$
\left|\varphi_{m n}\right|<\text { const }
$$

In this case the phase difference $\varphi(t)$ fluctuates around a constant value. After detection of all epochs of synchronization in the plot of $\varphi(t)$ their total duration is calculated and expressed in terms of total percentage.

it is possible that phase slips occur and the systems slip against each other by $2 \pi$ radians or a multiple thereof. If the coupling between the systems is strong then the phase difference rapidly changes - "jumps". The increase may be somewhat slower if the coupling is weak. In this regime, the phase difference is essentially unbounded and the criterion (3) cannot be fulfilled.

Several indices have been proposed to quantitatively measure phase synchronization. We Employed one of them that are based on the analysis of cyclic generalized phase difference:

$$
\varphi_{n m}(t)=\frac{\emptyset_{n m}(t)}{2 \pi}
$$

The synchronization index is the intensity of the first Fourier mode of the phase difference distribution

$$
\gamma_{n m}=\sqrt{\left|\left\langle\exp \left(\varphi_{n m}(t)\right)\right\rangle\right|}
$$

\section{RESULTS}

The sequence of R-R intervals and blood pressure for subject $\mathrm{S} 1$ clearly demonstrate the nonstationarity of the data.
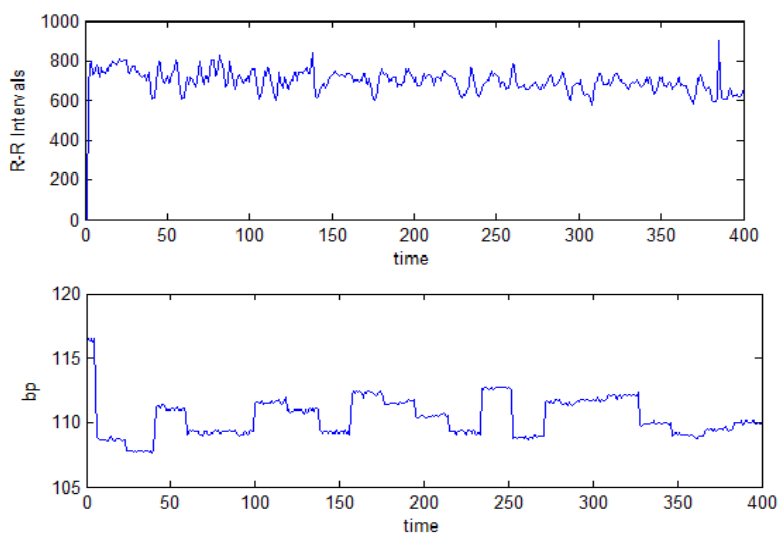

Fig1: (a) R-R intervals (b) Blood pressure

Once we get these signal then both of the signal will pass through the band pass filter with frequency band (0.05-0.15).
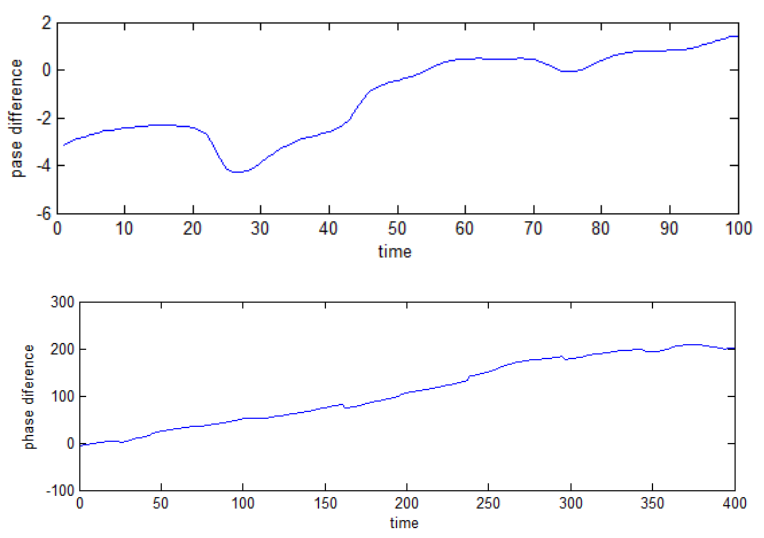

Fig 2: Generalized phase difference for subject A. Relative phase difference (a) shows some indication of 1:1 phase locking. For a comparatively short period of time i.e 20 s one can see plateaus in the plot of phase difference $\&$ time (b) The time dependence of phase difference remains approximately constant during the last $50 \mathrm{~s}$ for $3: 1$ phase locking.

As we already discuss when phase difference is almost constant for that period of time than ECG and BP are synchronized. From fig (2.a) we clearly see that there are one epochs in which phase difference is constant and period of synchronization is 20 s for $1: 1$ phase locking.

phase difference is constant and period of synchronization is 50s for 3:1 phase locking. 


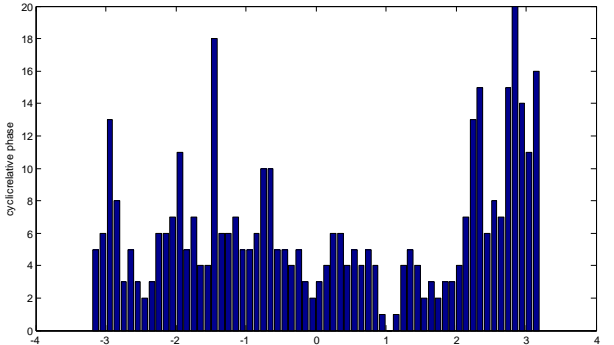

Fig 3: Cyclic Relative phase

With the help of cyclic relative phase we are clearly able to observe the synchronization because histogram will increase and then decrease in synchronization.

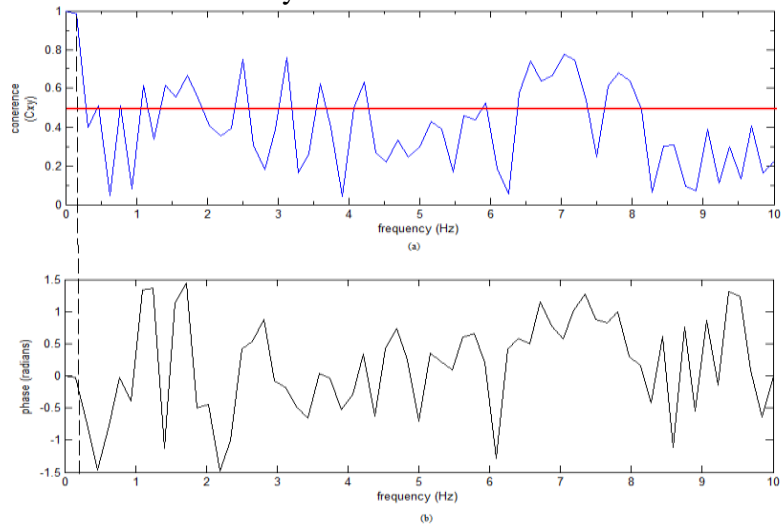

Fig3: (a) coherence (b) coherence-phase

Fig 4(a) \& 4(b) shows the coherence \& coherence phase plot between ECG \& BP. In (a) red line shows the reference value 0.5 , as we know that the two signal are coherent only when MSC having value $>0.5$. Both the signals are highly coherent and at frequency around $0.2 \mathrm{~Hz}$ we get max coherence (.94) value. At the same frequency $0.2 \mathrm{~Hz}$ from phase plot we clearly see that, the value of phase is negative so ECG will lag Behind BP.

For all other subject the phase synchronization is high in higher 3:1 phase locking. It can also be analyzed with the help of Table1 in which the value of synchronization index conform the result which were depicted by above graph (Fig2)

Table 1. Table shows the synchronization indexes for different subjects

\begin{tabular}{|c|l|l|}
\hline subject & $\gamma(\mathbf{3 : 1 )}$ & $\gamma(\mathbf{1 : 1 )}$ \\
\hline S1 & 0.548 & 0.343 \\
\hline S2 & 0.656 & 0.436 \\
\hline S3 & 0.887 & 0.543 \\
\hline S4 & 0.905 & 0.632 \\
\hline S5 & 0.764 & 0.465 \\
\hline S6 & 0.532 & 0.231 \\
\hline S7 & 0.683 & 0.324 \\
\hline S8 & 0.786 & 0.561 \\
\hline
\end{tabular}

\section{CONCLUSION}

Interaction is studied between the oscillating processes human heart and arterial blood pressure with the help of phase synchronization. It is found that all the subjects show longer epochs of synchronization in 3:1 phase locking, and it can be conformed from table1. With help of coherence phase we are easily find which signal is leading and which is lagging

We have to be aware of an important issue: how can we be sure that these patterns of the relative phase indeed indicate Synchronization, and, respectively, underlying nonlinear dynamics? How reliable is this indication? There is no straight way to answer these questions so far. Actually, as synchronization is not a state, but a process of adjustment of Rhythms due to interaction, we cannot prove its existence if We do not have access to the system parameters and cannot Check experimentally that the synchronous state is stable towards variation of the parameter mismatch within certain Range. As we are not able to do such experiments on humans, the only way to get some confirmation but certainly not a proof of our conclusions is to make use of the fact that the data are non stationary. Synchronization can be also observed in heart transplant subjects. These subjects have no direct neural regulation of the heart rate by ANS; therefore, in this case, some other mechanisms are responsible for the locking phenomenon.

\section{ACKNOWLEDGMENTS}

We gratefully acknowledge valuable discussions with faculty members Deptt. Of instrumentation and control engineering NIT Jlandhar.

\section{REFERENCES}

[1] A. S. Pikovsky, Sov. J. Commun. Technol. Electron. 30, 85(1985); E. F. Stone, Phys. Lett. A 163, 367 (1992); M. Rosenblum, A. Pikovsky, and J. Kurths, Phys. Rev.Lett. 76, 1804 (1996).versity of Washington.

[2] A. Pikovsky et al., Physica (Amsterdam) 104D, 219 (1997).

[3] U. Parlitz et al., Phys. Rev. E 54, 2115 (1996); C.M. Ticoset al., Phys. Rev. Lett. 85, 2929 (2000); E. Allaria et al.,Phys. Rev. Lett. 86, 791 (2001).

[4] I. Z. Kiss and J. L. Hudson, Phys. Rev. E 64, 046215 (2001).

[5] A. S. Pikovsky et al., in Handbook of Chaos Control, edited by H.G. Schuster (Wiley-VCH, Weinheim, Germany, 1999), pp. 305-328; A focus issue on phase synchronization in chaotic systems, edited by J.Kurths[Int. J. Bifurcation Chaos Appl. Sci. Eng. 7 (2000)].

[6] A Pikovsky, M. Rosenblum, and J. Kurths, Europhys.Lett. 34, 165 (1996).

[7] N. E. Huang, Z. Shen, S. R. Long, M. C. Wu, H. H. Shih, Q. Zheng, N.-C. Yen, C. C. Tung, and H. H. Liu, Proceedings: Mathematical, Physical and Engineering Sciences 454, 903 (1998).

[8] M. A. Zaks, E.-H. Park, M. G. Rosenblum, and J. Kurths, Phys. Rev. Lett. 82, 4228 (1999).

[9] R. Zou ${ }^{1}$, E. McCormack ${ }^{2}$, M. Egnor ${ }^{3}$, D. Kim ${ }^{4}$ M. Wagshul $^{5}$, B. MacDonald ${ }^{1}$, and J. R. Madsen ${ }^{1,4}$ “" Increased Phase Synchronization between Intracranial 
Pressure and Arterial Blood Pressure during Elevated Intracranial Pressure in Dogs", Proceedings of the 2005 IEEE Engineering in Medicine and Biology 27th Annual Conference Shanghai, China, September 1-4, 2005

[10] Aijun He, Xiaodong Yang, Xi Yang, Xinbao Ning, "phase synchronization in sleep electroencephalogram", IEEE International conference on complex medical engineering 2007.

[11] Gert Pfurtscheller*, Member, IEEE, Daniela S. Klobassa, Christof Altstätter, G ünther Bauernfeind, and Christa Neuper, " About the stability of the phase shifts between slow oscillation around $0.1 \mathrm{~Hz}$ in cardiovascular and cerebal system", IEEE Transactions on Biomedical Engineering Vol. 58. NO. 7, July 2011

[12] David M. Simpson*, Member, IEEE, Daniel A. Botero Rosas, and Antonio Fernando C. Infantosi, " Estimation of coherence between blood flow and spontaneous EEG Activity in neonates," IEEE Transaction on Biomedical Engineering, vol, 52, NO 5, May 2005.

[13] A.S.Pikovsky, M.G. rosenblum, G.V.Osipov, and J.Kurths, " Phase synchronization of chaotic oscillators by external driving," Physica D, vol, 104,pp,219-238, June 1997 